\title{
Studies on Development of Eye-Antennal Discs of Drosophila melanogaster in Tissue Culture, II. Effects of substances secreted from cephalic complexes upon eye-antennal discs of eye-mutant strains
}

\author{
Yoshihisa Fujio \\ Department of Biology, Faculty of Science, Osaka University, Osaka
}

Received September 15, 1961

It was suggested in the previous paper (Fujio, 1960) that the metamorphic hormone secreted from the cephalic complexes might be indispensable for growth and differentiation of the eye-antennal discs and that the cephalic complexes of several eye-mutant larvae might produce some unknown factors which promote growth and differentiation characteristic to several eye-mutant strains.

As suggested by Kuroda and Yamaguchi (1956) who used the tissue culture method, the metamorphic hormone of $B(B$ ar) larvae had different effects from that of Oregon-R larvae upon the growth and differentiation of the eye-antennal discs of $D$. melanogaster. Recently, it was also reported by Horikawa (1960) in his tissue culture experiments that the metamorphic hormone seemed to be different in quantity among eye-mutant strains of $D$. melanogaster. It is, however, known that there is no difference in the duration of larval and pupal periods among these eye-mutant strains.

It is very interesting to find whether the metamorphic hormone and other substances secreted from the cephalic complexes of these various strains have effects characteristic to their own strains upon growth and differentiation of the eye-antennal discs. To elucidate this problem, the eye-antennal discs were cultured in the media containing some substances secreted from the cephalic complexes which were previously cultured in the rotating tubes.

\section{Materials and Methods}

Oregon-R was used as $B, B(b b)-1, B$ (coiso), $B ; e^{11}$ (coiso), bar-3 (coiso), ey (coiso), and $D p$. The eye-antennal discs were cultured as described in the previous paper with some modifications. Aseptic third-instar larvae (about 95 hours old after hatching at $25^{\circ} \mathrm{C}$ ) were dissected under a binocular and the eye-antennal discs or the cephalic complexes were removed from the body in sterilized physiological salt solution. To obtain the media containing the substances secreted from the cephalic complexes, ten, twenty, forty, and sixty cephalic complexes were cultured respectively in $1.0 \mathrm{ml}$ of synthetic medium (K-6) at $25^{\circ} \mathrm{C}$ in rotating tubes (8 r.p.h.) for 24 hours and the cephalic complexes were removed from the media by decantation. The eye-antennal 
discs were cultured at $25^{\circ} \mathrm{C}$ in hanging-drops of these media. The microscopic examinations were performed on the eye-antennal discs cultured for 48 hours.

\section{Results}

Effects of the substances secreted from cephalic complexes of various strains on their own eye-antennal discs

To determine the effects of quantitative differences of the hormone secreted from the cephalic complexes upon growth and differentiation of the eye-antennal discs, the eye-antennal discs of various strains were cultured in the media in which ten, twenty, and forty cephalic complexes of their own strains had been previously cultured in rotating tubes for 24 hours. The results are shown in Table 1 . The substances

Table 1. Effects of substances secreted from cephalic complexes of various strains on their own eye-antennal discs

\begin{tabular}{|c|c|c|c|c|}
\hline \multicolumn{2}{|c|}{ Strains } & \multicolumn{3}{|c|}{ Number of cephalic complexes per m } \\
\hline Eye-antennal discs & Cephalic complexes & 10 & 20 & 40 \\
\hline Oregon- $\mathrm{R}$ & Oregon- $\mathrm{R}$ & - & ++ & ++ \\
\hline$B$ & $B$ & - & \pm & - \\
\hline$B(b b)-1$ & $B(b b)-1$ & - & \pm & - \\
\hline$B$ (coiso) & $B$ (coiso) & - & \pm & - \\
\hline$B ; e^{11}($ coiso $)$ & $B ; e^{11}($ coiso $)$ & - & \pm & - \\
\hline bar-3(coiso) & bar-3(coiso) & \pm & \pm & \pm \\
\hline$e y^{2}($ coiso $)$ & $e y^{2}$ (coiso) & + & + & \pm \\
\hline$D p^{*}$ & $D p^{*}$ & - & - & - \\
\hline
\end{tabular}

secreted from twenty and forty cephalic complexes of Oregon- $\mathrm{R}$ per $\mathrm{ml}$ of medium made its own eye-antennal discs display the complete growth and differentiation, although those from ten cephalic complexes failed to do so. This fact indicates that twenty or more cephalic complexes per $\mathrm{ml}$ of medium may provide the metamorphic hormone sufficient for growth and differentiation of the eye-antennal discs. On the other hand, the substances secreted from ten and forty cephalic complexes of $B, B$ (bb)-1, $B$ (coiso), $B ; e^{11}$ (coiso) per $\mathrm{ml}$ of medium made its own eye-antennal discs exert little effect on growth and differentiation. Those from twenty cephalic complexes of these strains produced a slight growth and differentiation. This suggests that the actions of some unknown factors promoting the growth and differentiation characteristic for the eye-mutants were lost by dilution. When the eye-antennal discs of bar3 (coiso) were cultured in the medium containing the substances secreted from its own 
ten, twenty, and forty cephalic complexes per $\mathrm{ml}$ of medium, their growth and differentiation were slightly observed. The substances secreted from ten and twenty cephalic complexes of $e y^{2}$ (coiso) per $\mathrm{ml}$ of medium produced more pronounced growth and differentiation of its own eye-antennal discs than those from forty cephalic complexes. It is interesting to note that the eye-antennal discs taken out from the mature third-instar larvae of $e \mathrm{y}^{2}$ (coiso) were morphologically different from those of other strains, that is, there was a knob on peripheral part of the former (Fig. 1). This knob seemed to affect growth and differentiation characteristic for this strain at later stages. Since this character is considered to be determined during the secondinstar larval period, the origin of this knob might be the second-instar. However, the eye-antennal discs of $D p$ cultured in the medium containing the substances secreted from its own ten, twenty, and forty cephalic complexes per $\mathrm{ml}$ of medium, showed no growth and differentiation.

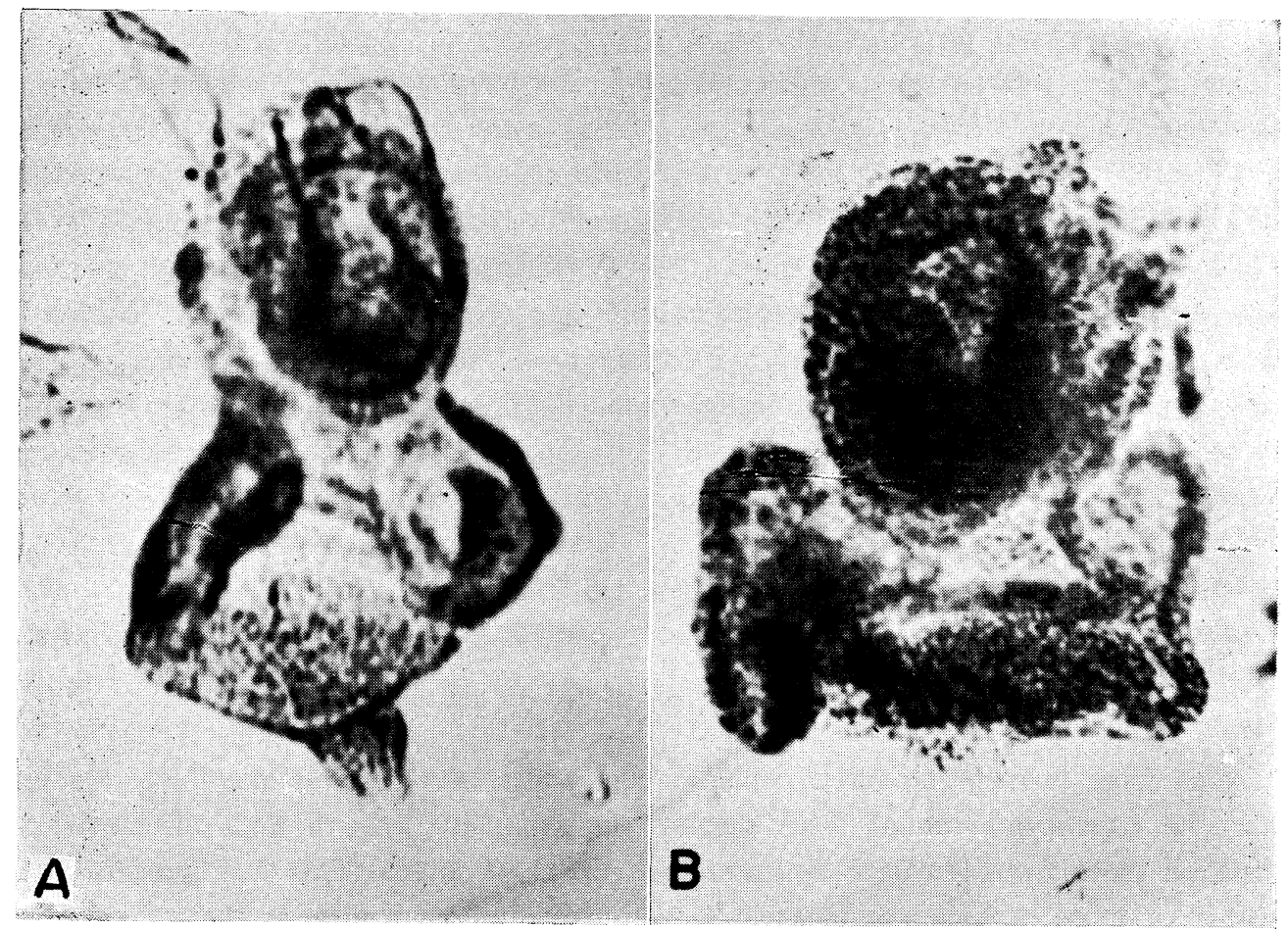

Fig. 1. $e y^{2}$ (coiso) eye-antennal discs cultured in the media containing the substances secreted from their own twenty cephalic complexes per $\mathrm{ml}$ of medium.

The eye-antennal discs taken out from the mature third instar larvae were morphologically different from those of other strains, that is, there was a knob on the peripheral part of the former. A: At the beginning B: After 48 hours

Effects of the substances secreted from cephalic complexes of various strains on eyeantennal discs of Oregon- $R$

In order to ascertain whether the metamorphic hormone or other substances 
secreted from the cephalic complexes of the eye-mutant larvae would promote the growth and differentiation characteristic for the eye-mutant strains, the experiments were carried out with the following results (Table 2).

Table 2. Effects of substances secreted from cephalic complexes of various strains on eye-antennal discs of Oregon- $R$

\begin{tabular}{c|c|c|c|c}
\hline \multicolumn{3}{c|}{ Strains } & \multicolumn{3}{|c}{ Number of cephalic complexes per ml } \\
\hline Eye-antennal discs & Cephalic complexes & 10 & 20 & 40 \\
\hline Oregon-R & Oregon-R & - & ++ & ++ \\
Oregon-R & $B$ & + & ++ & - \\
Oregon-R & $B(b b)-1$ & + & ++ & - \\
Oregon-R & $B($ coiso & + & ++ & - \\
Oregon-R & $B ; e^{11}($ coiso $)$ & \pm & ++ & - \\
Oregon-R & $b a r-3$ (coiso) & \pm & ++ & \pm \\
Oregon-R & $e y^{2}$ (coiso) & - & + & \pm \\
Oregon-R & $D p^{*}$ & - & + & - \\
\hline
\end{tabular}

* $\quad D p / \operatorname{In}(3 L) P, \operatorname{In}(3 R) C, S b$ e1(3)1

,,,- \pm+ and ++ signs show the degrees of growth and differentiation.

When the eye-antennal discs of Oregon- $\mathrm{R}$ were cultured in the medium containing the substances secreted from twenty cephalic complexes of $B, B(b b)-1, B$ (coiso), $B ; \mathrm{e}^{11}$ (coiso), or bar-3(coiso) per $\mathrm{ml}$ of medium, a marked growth and differentiation was observed, while ten or forty cephalic complexes per $\mathrm{ml}$ of medium produced a diminished or no growth and differentiation of the eye-antennal discs. In these cases, ten cephalic complexes per $\mathrm{ml}$ of medium seem to secrete the metamorphic hormone insufficient to support the growth and differentiation and forty cephalic complexes per $\mathrm{ml}$ of medium seem to provide other substances which promote the growth and differentiation characteristic for the eye-mutant strains. And twenty cephalic complexes per $\mathrm{ml}$ of medium seem to provide the metamorphic hormone sufficient for the growth and differentiation of the wild strain, and at the same time these might secrete diluted other substances in the medium. The substances secreted from the cephalic complexes of $e y^{2}$ (coiso) per $\mathrm{ml}$ of medium scarcely produced the growth and differentiation of the eye-antennal discs of Oregon- $\mathrm{R}$, although those from twenty and forty cephalic complexes per $\mathrm{ml}$ of medium produced a little growth and differentiation.

It is of interest that the growth and differentiation of the eye-antennal discs of Oregon- $\mathrm{R}$ was not brought about by the substances secreted from ten or forty cephalic complexes of $D p$ but done by those from twenty cephalic complexes.

These experiments described above demonstrate that the substances secreted from the cephalic complexes of $B, B(b b)-1, B$ (coiso), $B ; e^{11}$ (coiso), and $b a r$-3(coiso) seem to be similar to those of Oregon- $\mathrm{R}$ in their actions as the metamorphic hormone or the 
quantity of the former may be larger than those of the latter. The substances secreted from the cephalic complexes of $e y^{2}$ (coiso) or $D p$, however, seem to be similar to those of Oregon- $\mathrm{R}$ in their actions as the metamorphic hormone or the quantity of the former may be smaller than those of the latter. The cephalic complexes of these eye-mutant strains seem to provide other substances which have characteristic effects for their own strains upon the growth and differentiation of the eye-antennal discs. The chemical nature of these substances is unknown because of the difficulties to obtain them in large amounts from the cephalic complexes.

The differences of the growth and differentiation betweet the eye-antennal discs of eye-mutants and those of Oregon- $R$ shown by twenty cephalic complexes of eyemutants per $\mathrm{ml}$ of medium seem to be produced by these substances before the removal of the eye-antennal discs.

Effects of the substances secreted from cephalic complexes of Oregon-R on eye-antennal discs of various strains.

In the previous paper, it was indicated that the cephalic complexes of Oregon- $\mathrm{R}$ larvae had the effects of developing the eye of the wild type. To confirm it, the eyeantennal discs of various eye-mutants were cultured in the medium containing the substances secreted from the cephalic complexes of Oregon-R. As shown in Table 3,

Table 3. Effects of substances secreted from cephalic complexes of Oregon- $R$ on eye-antennal discs of various strains.

\begin{tabular}{|c|c|c|c|c|c|}
\hline \multicolumn{2}{|c|}{ Strains } & \multicolumn{4}{|c|}{ Number of cephalic complexes per ml } \\
\hline $\begin{array}{l}\text { Eye-antennal } \\
\text { discs }\end{array}$ & $\begin{array}{l}\text { Cephalic } \\
\text { complexes }\end{array}$ & 10 & 20 & 40 & 60 \\
\hline Oregon $\cdot \mathbf{R}$ & Oregon- $\mathrm{R}$ & - & ++ & $+r$ & + \\
\hline$B$ & Oregon-R & - & - & - & + \\
\hline$B(b b)-1$ & Oregon-R & - & - & - & + \\
\hline$B$ (coiso) & Oregon- $\mathrm{R}$ & - & - & \pm & + \\
\hline$B ; e^{11}($ coiso $)$ & Oregon- $\mathrm{R}$ & - & - & \pm & + \\
\hline bar-3(coiso) & Oregon- $\mathrm{R}$ & - & $+r$ & ++ & + \\
\hline$e y^{2}$ (coiso) & Oregon-R & - & + & + & + \\
\hline$D p^{*}$ & Oregon- $\mathrm{R}$ & - & - & - & - \\
\hline
\end{tabular}

the substances secreted from ten cephalic complexes of Oregon- $\mathrm{R}$ per $\mathrm{ml}$ of medium produced no growth and differentiation of the eye-antennal discs of any strains used in the present experiment. Twenty cephalic complexes of Oregon- $\mathrm{R}$ per $\mathrm{ml}$ of medium produced the growth and differentiation only in eye-antennal discs of bar-3 (coiso) and $e y^{2}$ (coiso) as well as in those of the wild type. Forty cephalic complexes 
of Oregon- $\mathrm{R}$ per $\mathrm{ml}$ of medium produced the growth and differentiation in the eyeantennal discs of bar-3 (coiso) and $e y^{2}$ (coiso) and a slight growth and differentiation in the eye-antennal discs of $B$ (coiso) and $B ; e^{11}$ (coiso). The above results suggest that the differences of the eye-antennal discs of several strains might be attributed to the other substances secreted from the cephalic complexes of these mutant strains before the removal of the eye-antennal discs. In other words, it is suggested that these substances secreted from the mutant strains might be different from one another among these strains. The substances secreted from sixty cephalic complexes of Oregon- $\mathrm{R}$ per $\mathrm{ml}$ of medium produced the growth and differentiation in the eyeantennal discs of all strains except $D p$. From these results, it would be concluded that the substances secreted from the cephalic complexes of Oregon-R contain the metamorphic hormone alone.

Effects of the cephalic complexes preincubated with urea on eye-antennal discs of their own strains.

As shown in the previous paper, urea seemed to affect the growth and differentiation of the eye-antennal discs through the cephalic complexes as a facet-increasing substance. To ascertain this fact the eye-antennal discs were cultured in the medium containing the substances secreted from the cephalic complexes which were previously cultured together with $1 \mathrm{mM}$ urea in rotating tubes. As control, the eye-antennal discs were cultured in the medium containing $1 \mathrm{mM}$ urea and the substances secreted from the cephalic complexes. Twently or forty cephalic complexes per $\mathrm{ml}$ of medium

Table 4. Effects of cephalic complexes preincubated with the urea on eye-antennal discs of their own strains

\begin{tabular}{c|c|c|c|c|c|}
\multicolumn{2}{|c|}{ Strains } & \multicolumn{2}{c|}{ Preincubation with urea } & \multicolumn{2}{c|}{ Preincubation without urea* } \\
$\begin{array}{c}\text { Eye-antennal } \\
\text { discs }\end{array}$ & $\begin{array}{c}\text { Cephalic } \\
\text { complexes }\end{array}$ & 20 C.C. & 40 C.C. & 20 C.C. & 40 C.C. \\
\hline $\begin{array}{c}\text { Oregon-R } \\
\text { Oregon-R }\end{array}$ & + & ++ & + & ++ \\
$B$ & $B$ & \pm & + & - & - \\
$B(b b)-1$ & $B(b b)-1$ & \pm & + & - & - \\
$B($ coiso $)$ & $B($ coiso & + & ++ & - & - \\
$B ; e^{11}($ coiso $)$ & $B ; e^{11}($ coiso $)$ & + & ++ & - & - \\
$b a r-3($ coiso $)$ & $b a r-3($ coiso $)$ & + & ++ & - & - \\
$e y^{2}$ coiso $)$ & $e y^{2}$ (coiso & + & ++ & \pm & - \\
$D p^{* *}$ & $D p^{* *}$ & - & - & - & - \\
\hline
\end{tabular}

C.C.: Number of cephalic complexes per $\mathrm{ml}$ of medium.

*: After adding $1 \mathrm{mM}$ urea in media containing substances from cephalic complexes, eyeantennal discs were cultured.

**: $\quad \operatorname{Dp} / \operatorname{In}(3 L) P, \operatorname{In}(3 R) C, S b$ e1(3)1

,,,- \pm+ and ++ signs show the degrees of growth and differentiation. 
were used in this experiment. The substances from the cephalic complexes preincubated without urea produced no growth and differentiation of the eye-antennal discs of the mutant strains, whereas the substances preincubated with cephalic complexes and urea could produce the growth and differentiation of eye-antennal discs of all strains except $D p$ (Table 4).

This fact indicates that urea does not affect directly the eye-antennal discs but it affects the cephalic complexes and subsequently the eye-antennal discs.

\section{Conclusion and Summary}

The eye-antennal discs taken out from the mature-third-instar larvae (about 95 hours old after hatching at $25^{\circ} \mathrm{C}$ ) were cultured in the medium containing the substances secreted from the cephalic complexes which were previously cultured in rotating tubes.

The substances secreted from twenty or more cephalic complexes of Oregon- $R$ per $\mathrm{ml}$ of medium produced the complete growth and differentiation of its own eyeantennal discs, although those from ten cephalic complexes per $\mathrm{ml}$ of medium failed to do so. The substances secreted from sixty cephalic complexes of Oregon- $\mathrm{R}$ per $\mathrm{ml}$ of medium produced the growth and differentiation of the eye-antennal discs of all strains except $D p$, which are similar to that of the wild type.

The substances secreted from ten and forty cephalic complexes of $B, B(b b)-1$, $B$ (coiso), and $B ; e^{11}$ (coiso) per $\mathrm{ml}$ of medium produced a diminished or no growth and differentiation in the eye-antennal discs of their own strains and of Oregon-R. Those from twenty cephalic complexes per $\mathrm{ml}$ of medium produced a slight growth and differentiation in its own eye-antennal discs and a marked growth and differentiation in the eye-antennal discs of Oregon- $R$. This fact indicates that the cephalic complexes of $B$ strains might secreted other substances $-B$ substances-, which have the characteristic effects of the growth and differentiation of the eye-antennal discs of $B$ strains, other than those of metamorphic hormone. In the case of bar-3(coiso), a substance resembling $B$ substance seem to be secreted from the cephalic complexes.

The eye-antennal discs taken out from the third-instar larvae of $e y^{2}$ (coiso) were morphologically different from those of the other strains, that is, a knob on their peripheral part was observed. The substances resembling $B$ substance were also secreted from the cephalic complexes of $e y^{2}$ (coiso) even in the third-instar larval period. The substances secreted from twenty cephalic complexes of $D p$ per $\mathrm{ml}$ of medium could produce the growth and differentiation of the eye-antennal discs of Oregon- $\mathrm{R}$, in their own forms.

$1 \mathrm{mM}$ urea had the effects as the facet-increasing substance on the eye-antennal discs of eye-mutant strains through the cephalic complexes. This fact suggests that urea seems to inhibit the secretion of $B$ or related substances from the cephalic complexes of various eye-mutant strains.

As the results of these facts, it may be concluded that the genic action operates in the secretion of $B$ or related substances which promote the growth and differentia- 
tion characteristic for the eye-mutant strains through the cephalic complexes of the eye-mutant larvae.

\section{Acknowledgement}

The author wishes to express his hearty thanks to Prof. H. Kikkawa for his advice and encouragement. Thanks are also due to Dr. Y. Kuroda for his kind guidance and criticism of this work.

\section{Literature cited}

Fujio, Y. 1960 Studies on the development of eye-antennal discs of Drosophila melanogaster in tissue culture, I. Effects of the facet-increasing substances upon the growth and differentia. tion of eye-antennal discs. Jap. Jour. Genet. 35: 361-370.

Horikawa, H. 1960 Developmental-genetic studies of tissue cultured eye discs of D. melanogaster, II. Effects of the metamorphic hormone (cephalic complex) upon growth and differentiation of eye-antennal discs, and strain differences in relation to metamorphic hormone. Jap. Jour. Genet. 35: 76-83.

Kuroda, Y. and K. Yamaguchi 1956 The effects of the cephalic complex upon the eye discs of D. melanogaster. Jap. Jour. Genet. 31: 98-103. 